\title{
AN EFFECT OF LIGHT UPON THE CONTACT POTENTIAL OF SELENIUM AND CUPROUS OXIDE.
}

By E. H. Kennard and E. O. Dieterich.

HE increased electrical conductivity of selenium under illumination has been explained in terms of the electron theory by assuming that the light produced an increase in the number of free electrons, and this explanation is strongly supported by observations on the rate of decay of the conductivity, which suggest the recombination of ions according to familiar laws. It occurred to the authors that the increase in the number of electrons ought to result in a change of the contact potential under illumination, because the electrons would tend to diffuse away from the illuminated region and would therefore leave the selenium more positive than before. A search for this effect was accordingly made; the work was interrupted by the departure of one of the authors, but it seems worth reporting as a quick survey of a new field.

\section{Methods of Observation.}

Two methods of observation were used. The first was a condenser method for the measurement of quick changes in contact potential. The plate of selenium, which was about $5 \mathrm{~cm}$. square and 0.2 to $0.4 \mathrm{~mm}$. thick, was mounted between a sheet of gauze above and a copper plate below (cf. Fig. I), the plates being insulated from each other and when

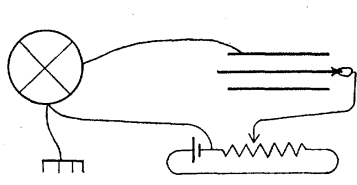

Fig. 1.

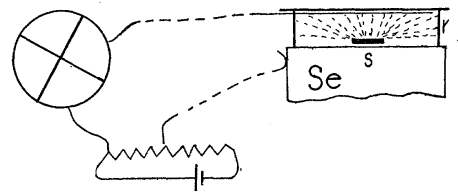

Fig. 2.

desired from earth by means of bits of amber or by supports tipped with sealing-wax. Any one of the three plates could be connected to an electrometer giving $3,000 \mathrm{~mm}$./volt or to an improvised potentiometer, contact with the selenium being made by means of a wire clip; thus any deflection of the electrometer due to a change in the potential of the selenium surface could be nullified by adjusting the potentiometer and the change was thus determined. Readings were usually taken in pairs, 
the light being thrown on and then off; the two readings generally differed by I0-20 per cent. but the means of successive pairs agreed mostly within a few per cent.

The second method was an adaptation of the ionization method of measuring contact potentials. An "ion contact" capsule (Fig. 2) was made by coating a copper strip $s$ on top with polonium and mounting it in a fiber ring $r$ so that it would clear when the ring lay upon a plane surface. The ring was closed on top by a sheet of mica, and just below this was mounted a sheet of brass gauze, one strand of which protruded through the ring and was connected to the electrometer. The mica was of sufficient thickness to stop $\alpha$-particles and all cracks were closed with soft wax, while the arrangement of the parts prevented $\alpha$-particles from striking the selenium. The ring was about $12 \mathrm{~mm}$. in diameter and $3 \mathrm{~mm}$. high.

A null method was employed as suggested by Fig. 2. The equalization of potential between gauze and specimen was only about half effected in a minute, corresponding to a resistance of some $\mathrm{IO}^{13} \mathrm{ohms}$, but when the sensitiveness of the electrometer was increased to $18,000 \mathrm{~mm}$./volt by the use of a concave lens in front of the scale the adjustment of the potentiometer within a millivolt could be made in less than a minute.

The specimen was illuminated by a 400-watt tungsten lamp placed some $30 \mathrm{~cm}$. away and focused roughly upon it by means of a large lens, a glass dish containing $2 \mathrm{~cm}$. of water being usually interposed to minimize heating effects. Electrostatic screening was carefully attended to, the light being admitted through a sheet of coarse gauze.

\section{Photo-Electromotive Effect in Selenium.}

Various connections of the plates in the condenser arrangement were employed, the selenium being connected either to the electrometer or to the potentiometer or left insulated; while the ion contact capsule was used chiefly on a I2-mm. cube of selenium. All the results consistently indicated that illumination suddenly made the surface of the selenium more negative, instead of more positive as anticipated. The effect on the plate was usually not far from - o.I volt, the deflections in the absence of compensation being of the order of $20 \mathrm{~mm}$. Shading the contact on the selenium had no appreciable effect. On the cube mean values of - o.I52, - 0.176 volt were obtained for a certain face on different days, while later, after washing thoroughly with chloroform, the effect was - 0.I29 on the same face and - 0.I25 volt on the opposite face. The latter observations indicate a variation with.the state of the surface, which we should rather expect if the phenomenon is a change in the contact potential. 
An E.M.F. in a closed circuit containing selenium was looked for repeatedly but without appreciable success. In one case two brass clips were placed on the selenium plate, each touching the plate on one (but not the same) side and insulated from the other surface by a bit of paper. When one clip was now connected to the electrometer and the other to earth, illumination produced a minute deflection that was consistent as to sign but amounted only to $.3 \mathrm{~mm}$. (o.I mv.); the sign was reversed by shading the upper contact. These minute residual deflections may very well be due to a heating effect, for the thermo-electric power of selenium is quite large. It is to be noted however that the actual point of contact between the selenium and the metal was not here appreciably illuminated.

A natural suspicion is that the effect might be due to photo-electric emission from the gauze above the selenium. But aside from the question of quantitative sufficiency, this explanation seems to be ruled out completely by these two facts: first, the effect is sudden and yet no steady current persisted under continuous illumination even when all potentials were kept the same by adjusting the potentiometer; secondly, the effect at once reverses when the light is removed.

The conclusion seems therefore a safe one that the observed effect is due to a sudden change produced by illumination in the contact potential of the selenium surface. In other words, between more and less strongly illuminated selenium there exists what might be called a "photo-electromotive force" tending to drive positive electricity away from the illuminated region.

\section{VARIATION WITH INTENSITy OF THE Light.}

The variation of the effect with the intensity of illumination was investigated briefly by varying the voltage on the lamp, using the polonium capsule and the selenium cube. An approximate calibration of the lamp was made in terms of an arbitrary standard.

A high degree of sensitiveness to faint light was at once apparent, for a change in potential of about $3 \mathrm{mv}$. was produced with only $\mathrm{I} 3$ volts on the lamp (normal, I Io); the illumination on the selenium must have been only about 0.0 I candle-foot. Yet at 25 volts, or 2-3 candle-feet, the effect had increased only to $35 \mathrm{mv}$. From this point on a series of readings was taken, the light being thrown on and off at $2 \mathrm{~min}$. intervals while its intensity was varied to full value and back. The photoelectromotive potentials are plotted against lamp voltages in Fig. 4, curve $I$, while in Fig. 3 the corresponding mean values are plotted on logarithmic scale against the intensity of the light. Something like an 
approach to saturation is evident, for the last light intensity is 2,000 times the first while the photo-electromotive effect is only increased by a factor of 3.6 ; but the logarithmic curve shows a persistent slow increase proportional to about the fifth or sixth root of the intensity.

Closely similar results were obtained when the light was kept on continuously (except for a short break while changing connections). In this case five minutes was allowed between readings, as a previous test had indicated that a fairly steady equilibrium was thus attained. The results are shown in Fig. 4, curve $I I$, the potential of the selenium relative to the gauze in the capsule being plotted against lamp voltage.

Fatigue, Drift and Time Lag.

Curve $I$ in Fig. 4 shows clear evidence of fatigue with respect to repetition, for the later readings taken after the lapse of 4-28 minutes are all 2-5 mv. smaller. A similar difference between readings taken in immediate succession was frequently noticed. Fatigue with respect to

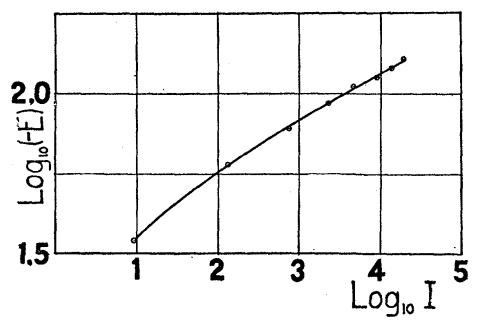

Fig. 3.

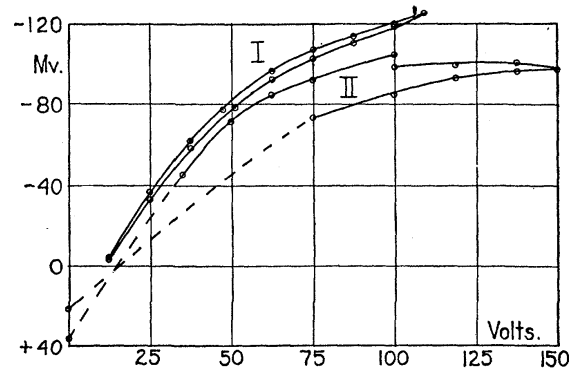

Fig. 4.

continuation, also, is indicated by curve $I I$, Fig. 4. The difference in the initial and final dark readings points to a drift of the dark potential toward the negative, yet during the strong illumination the potential was evidently drifting toward the positive: the most natural explanation is that the negative displacement due to illumination tended to decrease with time. Such a drift of the dark potential was frequently noticed; it probably accounted for an apparent hysteresis of 10-20 per cent. that was often observed between the on- and off-readings.

If any time lag occurred when the light was thrown on, it was a matter of seconds only and escaped detection. On the other hand, the change in potential seemed usually to.overshoot and then to settle back in the course of several minutes to an equilibrium value.

The Contact Potential of Selenium.

Several comparisons of the contact potential of selenium with that of freshly sandpapered copper were made. 
Measurements on two different plates by the usual variable condenser method gave, in the dark, $-0.34,-0.45$ volt, resp. Two faces of the cube were found to be at -.27 and -.36 , resp., relative to the gauze in the polonium capsule, while on the same day the latter was observed to be -.099 relative to copper, giving for the selenium -0.37 and -0.46 , resp. Thus the contact potential of selenium crystallized in mass is not far from -0.4 volt in the dark relative to freshly sandpapered copper, changing under moderate to intense illumination by about - o.I volt.

\section{Photo-Electromotive Effect in Cuprous Oxide.}

Interesting results were obtained on a plate of cuprous oxide, which is also light-sensitive as regards resistance. The plate was about $2.5 \mathrm{~cm}$. square and I mm. thick, and was prepared by heating a copper plate at a bright red heat for 48 hours and then dissolving off the surface layer of cupric oxide with aqua regia. The time allowed proved insufficient, however, so that the plate contained a layer of metallic copper down the middle.

The polonium capsule was placed on top of the plate and two clips were attached, one insulated with paper above and the other below. By connecting one clip to the electrometer and the other to the potentiometer it was found that an E.M.F. (voltaic?) of some $20 \mathrm{mv}$. existed between these two points; under illumination this E.M.F. increased by

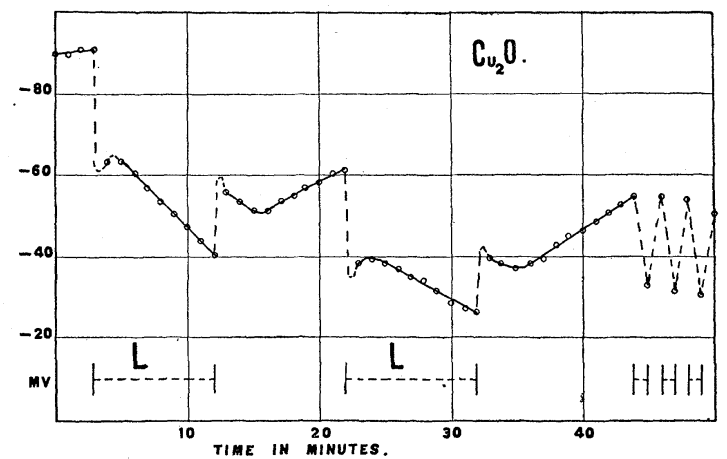

Fig. 5.

$7 \mathrm{mv}$. when the top contact was unshaded but decreased by the same amount when the contact was shaded. When the capsule was connected to the electrometer and one of the clips to the potentiometer, illumination made the surface under the capsule become more positive by 20 to $28 \mathrm{mv}$.

As in selenium, the initial effect seemed to overshoot and to be followed by a moderate reaction. There was also a decided drift of the potential 
in the dark, sometimes in one direction, sometimes in the other, but a true hysteresis was not apparent. A series of readings of the potential at intervals of a minute is shown in Fig. 5, the dotted line marked $L$ showing the periods of illumination. Apparently illumination produçes, besides the sudden effect, a slow drift of potential followed by recovery in the dark but lagging by $2-3$ minutes; this may be a temperature effect. The curve shows decided fatigue with respect to continuation of illumination, the return being practically complete if made at once but amounting to only some 60 per cent. after ten minutes of illumination. Fatigue with respect to repetition does not appear here, but in some of the other readings it was evident to the extent of some Io per cent.

\section{Conclusion.}

The negative sign of the effect in selenium disposes at once of the diffusion of electrons as a possible cause. A tempting hypothesis is that the light modifies the molecules in such a way as to diminish the potential energy of the electrons in the intermolecular spaces, and that this results in an increase of the number of free electrons, partly at the expense of the surrounding atoms of selenium and partly at the expense of other bodies with which it is in contact. This would explain both the increased conductivity and the change toward the negative in the contact potential. But the lack of proportionality between the two effects and especially the apparent occurrence of saturation in the latter seem to be fatal

objections to this hypothesis. Apparently the two effects are due to independent causes.

The experimental results may be summarized as follows:

I. The contact potential of selenium changes under illumination by an amount exceeding a millivolt for o.or candle-foot and tending to show saturation at a value of about - o.I volt under strong illumination. The phenomenon is complicated by fatigue effects and a slow drift of the potential with time.

2. No E.M.F. is produced however in a closed circuit.

3. The contact potential of selenium relative to freshly sandpapered copper is about -0.4 volt in the dark.

4. The surface potential of a plate of cuprous oxide changed under illumination by about $25 \mathrm{mv}$; but the plate also contained a small voltaic (?) E.M.F. which varied under illumination.

The authors take pleasure in acknowledging a debt to Professor A. F. Kovarik for his kindness in preparing the polonium deposit for the ion contact capsule.

UNIVERSity OF MinNesota, August, ror6. 